\title{
The case for routine goals-of-care documentation
}

\author{
Christopher Yarnell, ${ }^{1}$ Robert Fowler ${ }^{1,2}$
}

${ }^{1}$ Department of Medicine, Sunnybrook Health Sciences Centre and the University of Toronto, Toronto, Canada ${ }^{2}$ Interdepartmental Division of Critical Care Medicine, University of Toronto, Toronto, Canada

\section{Correspondence to}

Dr Robert Fowler, Sunnybrook Health Sciences Centre, Room H468, 2075 Bayview Avenue, Toronto, Ontario M4N 3M5, Canada; rob.fowler@ sunnybrook.ca

Accepted 20 November 2015 Published Online First 15 December 2015

\section{CLinked}

- http://dx.doi.org/10.1136/ bmjqs-2015-004567

\section{CrossMark}

$$
\begin{aligned}
& \text { To cite: Yarnell C, Fowler R. } \\
& \text { BMJ Qual Saf 2016;25:647- } \\
& \text { 648. }
\end{aligned}
$$

The article by Heyland et $a l^{1}$ reveals a $37 \%$ prevalence of discordance between patient preferences and hospital chart documentation for cardiopulmonary resuscitation (CPR) and other lifesustaining treatments. This high prevalence of discordance represents significant potential for avoidable, preventable harm. These findings demand that effective goals-of-care discussions and documentation should become as routine as eliciting and documenting medication allergies.

This study at 16 Canadian hospitals convincingly depicts inadequate documentation of goals of care. The authors calculated error rates using interviews with 500 hospitalised patients and 408 family members of hospitalised patients to learn their goals of care. They compared these goals with the documented goals of care in the patient orders and uncovered a $35 \%$ rate of potential overtreatment, defined as the proportion of patients who preferred to forego CPR while the chart lacked corresponding orders. The authors also measured a $2 \%$ rate of potential undertreatment, defined as the proportion of patients who preferred CPR while the chart had orders for no CPR. In other words, these patients would be at risk for undergoing no resuscitative efforts despite a preference for doing so.

Heyland and colleagues have applied a novel and provocative nomenclature of 'medical error' to discordance in patient goals of care and documentation of these goals. We agree with the authors' decision to label these discrepancies as medical errors. It may be tempting to downplay the importance of these errors because, at first glance, the potential for harm appears small. Only one patient received CPR during their index hospitalisation. And, for patients with discordant preference documentation, clinical deterioration may precipitate a reassessment of the goals of care before over (or under) treatment occurs. However, some patients deteriorate quickly or even suddenly, in which case goals-of-care discussions cannot occur and pre-existing orders in the chart prevail. In at least some of these discrepant cases, the erroneous documentation of patients' actual preferences carries substantial risk. Therefore, we think it reasonable to label such discordance a medical error (or even a potential adverse event) irrespective of harm or near-miss attribution.

In this study, only $84(17 \%)$ patients preferred CPR and were ordered to receive it. Assuming that (1) the 242 (48\%) patients who had 'No CPR' orders would not receive CPR and (2) the chance of CPR is uniformly distributed across the remaining patients, then these data imply that $67 \%$ of CPR episodes could be performed on patients who did not want to receive it. A recent review of CPR data from the USA showed an inhospital CPR rate of 2.95 per 1000 patient admissions. ${ }^{2}$ This implies a rate of about two cases of inappropriate CPR per 1000 patients. That a vast majority of patients in this study preferred not to have CPR challenges the popular notion that CPR should definitely be a 'default' plan of care for patients in our hospitals.

The authors were unable to enrol significant numbers of patients who did not speak English, patients with cognitive impairment or patients who refused to speak about goals of care. Goals-of-care discussions in those groups may be complex and even more prone to discordance. In addition, the majority of patients endorsed a protestant religious background. A population with more religious diversity might have a different baseline rate of preference for no CPR and thereby decrease the error rate. Despite these limitations, the discordance observed by Heyland and colleagues strongly suggests the need for improvement.

Consider the parallel with allergy. The rate of cutaneous allergic drug reactions in 
hospital is $1.8-4.2$ cases per 1000 patients. $^{3}$ It is considered inappropriate to prescribe medication without first ascertaining a patient's allergies. Medical students learn to ask about allergies at every new patient encounter. Electronic medical records provide alerts when allergies are not documented. Many patients have straightforward allergy histories, but some patients require a nuanced approach and are assessed by specialists. Similarly, it is inappropriate-indeed a potential medical error-to prescribe a plan of treatment without first ascertaining a patient's goals of care. Medical training should emphasise and teach the importance of ensuring appropriately informed goals of care are known for all patients. Electronic medical records could provide alerts when goals of care are not documented or discordant with prior encounters.

Making goals of care a routine element of the clinical encounter will not resolve all problems in goals-of-care documentation. Incomplete understanding between patients and physicians may persist. Some physicians may lack expertise in specific details about life-sustaining therapies or techniques for goals-of-care discussion. Some patients may change their preferences without updating their healthcare team. Other patients may be uncomfortable with the discussion of goals of care and require a careful approach to avoid damaging the physician-patient relationship. Several tools and guides exist to facilitate effective goals-of-care discussions. ${ }^{4}$
The first step in improving the quality of care is to investigate and document current practice.

The work by Heyland et al demonstrates the current discrepancy between our patients' preferences and our documentation of those preferences. Incorporating goals-of-care discussions into the clinical encounter as a routine element may decrease this discrepancy and promote the communication, partnership and clinical decision-making that our patients deserve.

Competing interests None declared.

Provenance and peer review Commissioned; internally peer reviewed.

\section{REFERENCES}

1 Heyland DK, Ilan R, Jiang X, et al. The prevalence of medical error related to end of life communication in Canadian hospitals: results of a multi-center observational study. BMJ Qual Saf 2016;25:671-9.

2 Kazaure HS, Roman SA, Sosa JA. Epidemiology and outcomes of in-hospital cardiopulmonary resuscitation in the United States, 2000-2009. Resuscitation 2013;84:1255-60.

3 Thong BY-H, Tan T-C. Epidemiology and risk factors for drug allergy. Br J Clin Pharmacol 2011;71:684-700.

4 You JJ, Fowler RA, Heyland DK; Canadian Researchers at the End of Life Network (CARENET). Just ask: discussing goals of care with patients in hospital with serious illness. CMAJ 2014; $186: 425-32$. 